\title{
Lattice Bhatnagar-Gross-Krook model for the Lorenz attractor
}

\author{
Guangwu Yan ${ }^{\mathrm{a}, \mathrm{b}, *}$, Li Yuan $^{\mathrm{a}}$ \\ a LSEC, Institute of Computational Mathematics, Academy of Mathematics and System Sciences, \\ Chinese Academy of Sciences, Beijing 100080, PR China \\ b Section of Mechanics, Department of Mathematics, Jilin University, Changchun 130023, PR China \\ Received 15 October 1999; received in revised form 12 December 2000; accepted 10 January 2001 \\ Communicated by U. Frisch
}

\begin{abstract}
In this paper, we propose a new lattice Bhatnagar-Gross-Krook model for simulation of the Lorenz attractor. The new model is based on a nonlinear diffusion-reaction system. We formulate a lattice Bhatnagar-Gross-Krook model for the nonlinear diffusion-reaction system by using a method of higher moments of the lattice Boltzmann equation. As a special case where the diffusion effect disappears, we get the Lorenz equation. In the model, we obtain a series of lattice Boltzmann equations at different time scales, and the conservation law at time scale $t_{0}$. The equilibrium distribution functions are simpler than those for the standard lattice Boltzmann model. The numerical examples show that the method can be used to simulate the Lorenz equation. (C) 2001 Elsevier Science B.V. All rights reserved.
\end{abstract}

Keywords: Lattice BGK model; Multiscale technique; Lorenz attractor

\section{Introduction}

Since its precursor - the lattice gas automaton (LGA) - was proposed 10 years ago as a useful computational fluid dynamics (CFD) technique [1], the lattice Bhatnagar-Gross-Krook (BGK) method has emerged as a promising approach for simulation of complex flows [2-4]. Unlike traditional CFD methods which solve macroscopic equations, the lattice BGK method simulates fluid flow based on microscopic models or mesoscopic kinetic equations. This intrinsic feature enables the lattice BGK method to incorporate easily essential physics at microscopic or mesoscopic level. Several lattice BGK models for simulation of diffusion-reaction system have been proposed in the past several years [5-9]. Although each of the above lattice BGK diffusion-reaction models was built on different physical pictures and has a quite different appearance, a recent study by Yan et al. [10] showed that all of them have an origin in the kinetic theory. Especially, all these models can be derived by using higher-order moment method with multiscale technique.

\footnotetext{
${ }^{*}$ Corresponding author. Present address: Section of Mechanics, Department of Mathematics, Jilin University, Changchun 130023, PR China. E-mail address: yan1118@mail.j1.cn (G. Yan).
} 


\section{Lattice BGK model}

\subsection{The definition of macroscopic quantity}

Consider a 1D or 2D model, we discretize the velocity of particles into $b$ directions, a lattice with unit spacing is used where each node has $b$ nearest neighbors connected by $b$ links. The particles velocity $\mathbf{e}_{\alpha}=\{0, c,-c\}, b=2$ for one-dimensional lattice; $\mathbf{e}_{\alpha}=\{(0,0),(c, 0),(0, c),(-c, 0),(0,-c)\}, b=4$ for two-dimensional lattice, here $\alpha=0,1, \ldots, b(\alpha=0$ is the rest particle).

Let us define $f_{\alpha}^{\sigma}$ to be the probability of finding a particle of species $\sigma$ at time $t$, at node $\mathbf{x}$, with velocity $\mathbf{e}_{\alpha}$. The density of species $\sigma$ is defined by

$$
u^{\sigma}(\mathbf{x}, \mathbf{t})=\sum_{\alpha} \mathbf{f}_{\alpha}^{\sigma}(\mathbf{x}, \mathbf{t})
$$

The lattice BGK Boltzmann equation reads as

$$
f_{\alpha}^{\sigma}(\mathbf{x}+\Delta \mathbf{x}, t+\Delta t)-f_{\alpha}^{\sigma}(\mathbf{x}, t)=\Omega_{\alpha}^{\sigma}(\mathbf{x}, t)+\omega_{\alpha}^{\sigma}(\mathbf{x}, t) .
$$

In the case of standard lattice Boltzmann [2-4], the term $\omega_{\alpha}^{\sigma}=0$. For $\Omega_{\alpha}^{\sigma}$, we use the BGK approximation, i.e. $\Omega_{\alpha}^{\sigma}=-(1 / \tau)\left[f_{\alpha}^{\sigma}(\mathbf{x}, t)-f_{\alpha}^{\sigma, \text { eq }}(\mathbf{x}, t)\right]$, where the equilibrium distribution function, $f_{\alpha}^{\sigma, \text { eq }}$, depends on $\mathbf{x}$ and $t$ through the local density. $\omega_{\alpha}^{\sigma}$ is the additional term and $\tau$ is the single relaxation time factor.

As in the usual Chapman-Enskog expansion [12], we impose the following conservation condition on $f_{\alpha}^{\sigma}(\mathbf{x}, t)$ :

$$
\sum_{\alpha} f_{\alpha}^{\sigma, \mathrm{eq}}(\mathbf{x}, t)=u^{\sigma}(\mathbf{x}, t)
$$

\subsection{A series of lattice Boltzmann equations in different time scales}

Using $\varepsilon$ as the time step unit $\Delta t=\Delta x / c$ in physical unit, $\varepsilon$ can play the role of the Knudsen number [10,11], the lattice Boltzmann equation (2) in physical units is

$$
f_{\alpha}^{\sigma}\left(x+\varepsilon e_{\alpha}, t+\varepsilon\right)-f_{\alpha}^{\sigma}(x, t)=-\frac{1}{\tau}\left[f_{\alpha}^{\sigma}-f_{\alpha}^{\sigma, \mathrm{eq}}\right]+\omega_{\alpha}^{\sigma} .
$$

Making Taylor expansion of Eq. (4), and retaining terms up to $\mathrm{O}\left(\varepsilon^{5}\right)$ results in

$$
\begin{aligned}
f_{\alpha}^{\sigma}\left(x+\varepsilon e_{\alpha}, t+\varepsilon\right)-f_{\alpha}^{\sigma}(x, t)= & \varepsilon\left[\frac{\partial}{\partial t}+e_{\alpha} \frac{\partial}{\partial x}\right] f_{\alpha}^{\sigma}+\frac{\varepsilon^{2}}{2}\left[\frac{\partial}{\partial t}+e_{\alpha} \frac{\partial}{\partial x}\right]^{2} f_{\alpha}^{\sigma}+\frac{\varepsilon^{3}}{6}\left[\frac{\partial}{\partial t}+e_{\alpha} \frac{\partial}{\partial x}\right]^{3} f_{\alpha}^{\sigma} \\
& +\frac{\varepsilon^{4}}{24}\left[\frac{\partial}{\partial t}+e_{\alpha} \frac{\partial}{\partial x}\right]^{4} f_{\alpha}^{\sigma}+\mathrm{O}\left(\varepsilon^{5}\right)
\end{aligned}
$$

Next, the Chapman-Enskog expansion [12] is applied to $f_{\alpha}^{\sigma}$ under the assumption that the mean free path is of the same order of $\varepsilon$. Expanding $f_{\alpha}^{\sigma}$ about $f_{\alpha}^{\sigma,(0)}$

$$
f_{\alpha}^{\sigma}=\sum_{n=1}^{\infty} \varepsilon^{n} f_{\alpha}^{\sigma,(n)}=f_{\alpha}^{\sigma,(0)}+\varepsilon f_{\alpha}^{\sigma,(1)}+\varepsilon^{2} f_{\alpha}^{\sigma,(2)}+\varepsilon^{3} f_{\alpha}^{\sigma,(3)}+\varepsilon^{4} f_{\alpha}^{\sigma,(4)}+\cdots,
$$

where $f_{\alpha}^{\sigma,(0)}$ is $f_{\alpha}^{\sigma, \text { eq }}$. 
We discuss changes in different time scales, introduced as $t_{0}, \ldots, t_{3}$; thus

$$
t_{0}=t, \quad t_{1}=\varepsilon t, \quad t_{2}=\varepsilon^{2} t, \quad t_{3}=\varepsilon^{3} t,
$$

and

$$
\frac{\partial}{\partial t}=\frac{\partial}{\partial t_{0}}+\varepsilon \frac{\partial}{\partial t_{1}}+\varepsilon^{2} \frac{\partial}{\partial t_{2}}+\varepsilon^{3} \frac{\partial}{\partial t_{3}}+\mathrm{O}\left(\varepsilon^{4}\right) .
$$

Assume

$$
\omega_{\alpha}^{\sigma}=\varepsilon^{2} \phi_{\alpha}^{\sigma}
$$

Eq. (8) is an important assumption; its meaning is that reaction effect and diffusion one are at same time scale $\varepsilon^{2}$. In fact, if we choose $\omega_{\alpha}^{\sigma}=\varepsilon^{k} \phi_{\alpha}^{\sigma}$, where $k \neq 2$, then, derivation of the diffusion-reaction equations become very difficult. In this paper, we use $k=2$ to derive the series lattice Boltzmann equations as follows:

$$
\begin{aligned}
& \frac{\partial f_{\alpha}^{\sigma,(0)}}{\partial t_{0}}+e_{\alpha} \frac{\partial f_{\alpha}^{\sigma,(0)}}{\partial x}=-\frac{1}{\tau} f_{\alpha}^{\sigma,(1)}, \\
& \frac{\partial f_{\alpha}^{\sigma,(0)}}{\partial t_{1}}-\tau\left(1-\frac{1}{2 \tau}\right)\left(\frac{\partial}{\partial t_{0}}+e_{\alpha} \frac{\partial}{\partial x}\right)^{2} f_{\alpha}^{\sigma,(0)}=-\frac{1}{\tau} f_{\alpha}^{\sigma,(2)}+\phi_{\alpha}^{\sigma} \\
& \frac{\partial f_{\alpha}^{\sigma,(0)}}{\partial t_{2}}+(1-2 \tau)\left(\frac{\partial}{\partial t_{0}}+e_{\alpha j} \frac{\partial}{\partial x_{j}}\right) \frac{\partial f_{\alpha}^{\sigma,(0)}}{\partial t_{1}}+\left(\tau^{2}-\tau+\frac{1}{6}\right)\left(\frac{\partial}{\partial t_{0}}+e_{\alpha j} \frac{\partial}{\partial x_{j}}\right)^{3} f_{\alpha}^{\sigma,(0)} \\
& =-\frac{1}{\tau} f_{\alpha}^{\sigma,(3)}+(-\tau)\left(\frac{\partial}{\partial t_{0}}+e_{\alpha j} \frac{\partial}{\partial x_{j}}\right) \phi^{\sigma}\left(u^{\sigma}\right) .
\end{aligned}
$$

\subsection{The Lorenz equations}

Taking the summation in Eqs. (9) and (10) about $\alpha$, computing (9) $+(10) \times \varepsilon$, we get

$$
\frac{\partial u^{\sigma}}{\partial t}=\lambda^{\sigma} \varepsilon\left(\tau-\frac{1}{2}\right) \frac{\partial^{2} u^{\sigma}}{\partial x_{i} \partial x_{i}}+\varepsilon \phi^{\sigma}\left(u^{\sigma}\right)(b+1)+\mathrm{O}\left(\varepsilon^{2}\right) .
$$

To find the structure of the truncation error, we take $(9)+(10) \times \varepsilon+(11) \times \varepsilon^{2}$, and get

$$
\begin{aligned}
\frac{\partial u^{\sigma}}{\partial t}= & \lambda^{\sigma} \varepsilon\left(\tau-\frac{1}{2}\right) \frac{\partial^{2} u^{\sigma}}{\partial x_{i} \partial x_{i}}+\varepsilon \phi^{\sigma}\left(u^{\sigma}\right)(b+1)-2 \varepsilon^{2}\left(\tau^{2}-\tau+\frac{1}{6}\right) \lambda^{\sigma} \nabla^{2} \frac{\partial u^{\sigma}}{\partial t_{0}} \\
& -\tau \varepsilon^{2}(b+1) \frac{\partial \phi^{\sigma}}{\partial t_{0}}+\mathrm{O}\left(\varepsilon^{3}\right) .
\end{aligned}
$$

From Eq. (13), we get the truncation error as follows:

$$
R=-2 \varepsilon^{2}\left(\tau^{2}-\tau+\frac{1}{6}\right) \lambda^{\sigma} \nabla^{2} \frac{\partial u^{\sigma}}{\partial t_{0}}-\tau \varepsilon^{2}(b+1) \frac{\partial \phi^{\sigma}}{\partial t_{0}}+\mathrm{O}\left(\varepsilon^{3}\right) .
$$

The truncation error $R$ contains a dissipation term and an unsteady source term. Therefore, on second-level $\left(\varepsilon^{2}\right)$, the term $\partial u^{\sigma} / \partial t_{0}$ is dissipative with time. 
By choosing

$$
\begin{aligned}
& \sum_{\alpha} f_{\alpha}^{\sigma,(0)} e_{\alpha i}=0, \\
& \sum_{\alpha} f_{\alpha}^{\sigma,(0)} e_{\alpha i} e_{\alpha j}=\lambda^{\sigma} u^{\sigma} \delta_{i j},
\end{aligned}
$$

where $\lambda^{\sigma}=D_{\sigma} / \varepsilon\left(\tau-\frac{1}{2}\right)$, Eq. (12) becomes

$$
\frac{\partial u^{\sigma}}{\partial t}=D_{\sigma} \frac{\partial^{2} u^{\sigma}}{\partial x_{i} \partial x_{i}}+\psi^{\sigma}\left(u^{\sigma}\right)+\mathrm{O}\left(\varepsilon^{2}\right),
$$

where $\psi^{\sigma}\left(u^{\sigma}\right)=(b+1) \varepsilon \phi^{\sigma}\left(u^{\sigma}\right)$ is the source term of Eq. (12). We get the truncation error

$$
R=\mathrm{O}\left(\varepsilon^{2}\right) .
$$

The equilibrium distribution function $f_{\alpha}^{\sigma,(0)}$ can be expressed as follows:

$$
f_{0}^{\sigma,(0)}=u^{\sigma}-\frac{\lambda^{\sigma} D u^{\sigma}}{c^{2}},
$$
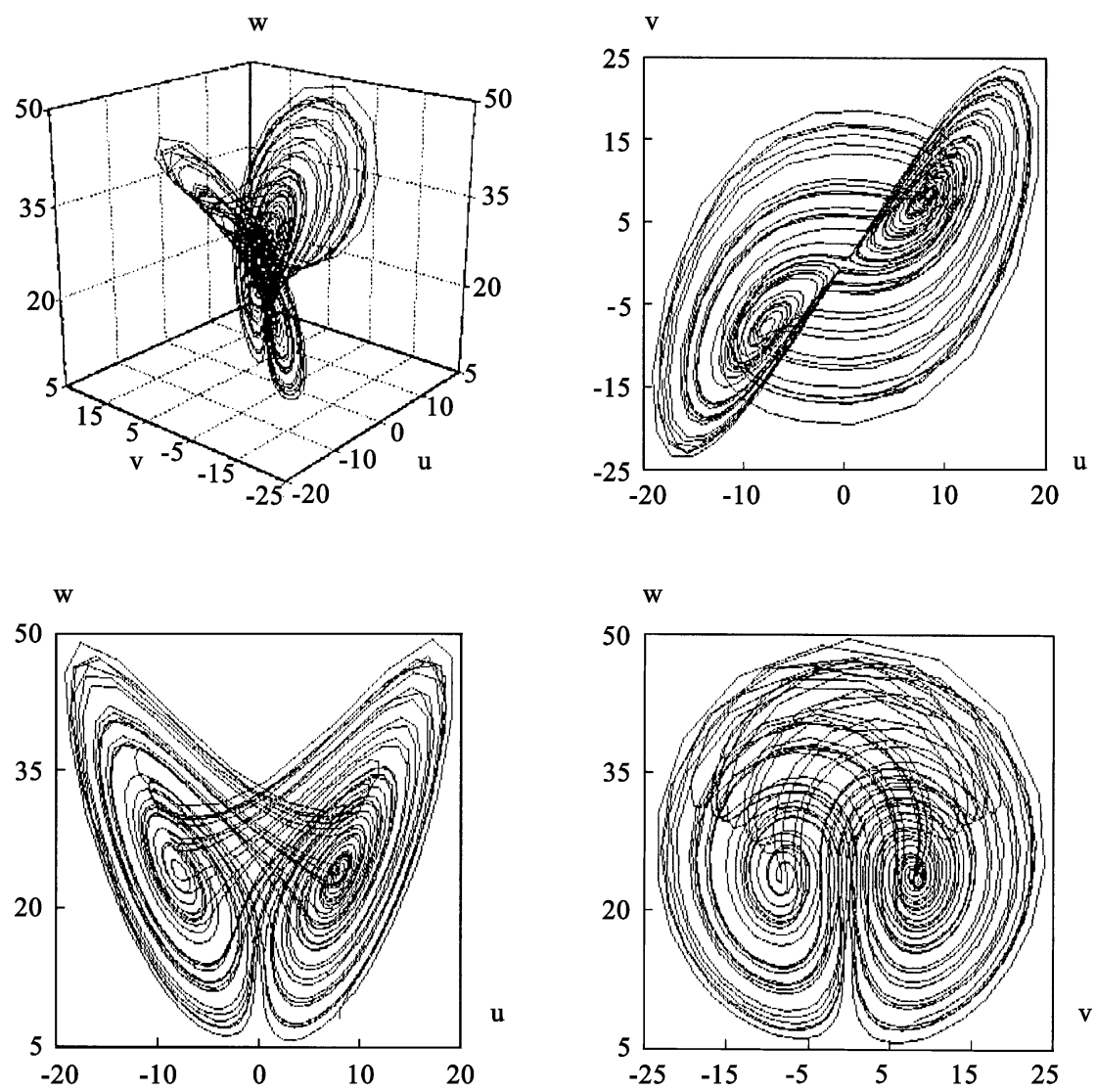

Fig. 1. Lorenz attractors of phase space at position $x=\frac{1}{2} N$. Parameters: $D_{1}=0.0, D_{2}=0.0, D_{3}=0.0 ; \xi=10.0, \eta=24.8, \zeta=\frac{8}{3}, c=10.0$, $1 / \tau=2$.2. Initial conditions: $\left.u\right|_{t=0}=\left.v\right|_{t=0}=\left.w\right|_{t=0}=8$.1. Lattice size: $N=200$. 


$$
f_{\alpha}^{\sigma,(0)}=\frac{\lambda^{\sigma} D u^{\sigma}}{b c^{2}}, \quad \alpha=1, \ldots, b .
$$

Using $M=3$ and $u^{1}=u, u^{2}=v, u^{3}=w$, from Eq. (17), we obtain the following diffusion-reaction equations:

$$
\frac{\partial u}{\partial t}=D_{1} \nabla^{2} u-\xi u+\xi v, \quad \frac{\partial v}{\partial t}=D_{2} \nabla^{2} v+\eta u-v-u w, \quad \frac{\partial w}{\partial t}=D_{3} \nabla^{2} w-\zeta w+u v .
$$

In order to get the Lorenz equations [13,14], we assign $\lambda^{\sigma} \rightarrow 0$ and $u=u(t), v=v(t), w=w(t)$, thus Eqs. (21) become the following form:

$$
\frac{\mathrm{d} u}{\mathrm{~d} t}=-\xi u+\xi v, \quad \frac{\mathrm{d} v}{\mathrm{~d} t}=\eta u-v-u w, \quad \frac{\mathrm{d} w}{\mathrm{~d} t}=-\zeta w+u v .
$$

\section{Numerical tests}

We select three values of $D_{\sigma}$ :

1. $D_{\sigma}=0.0$ which corresponds to Eq. (22). In this case, the equilibrium distribution functions are

$$
f_{0}^{\sigma}=u^{\sigma}, \quad f_{\alpha}^{\sigma}=0, \quad \alpha=1, \ldots, b .
$$
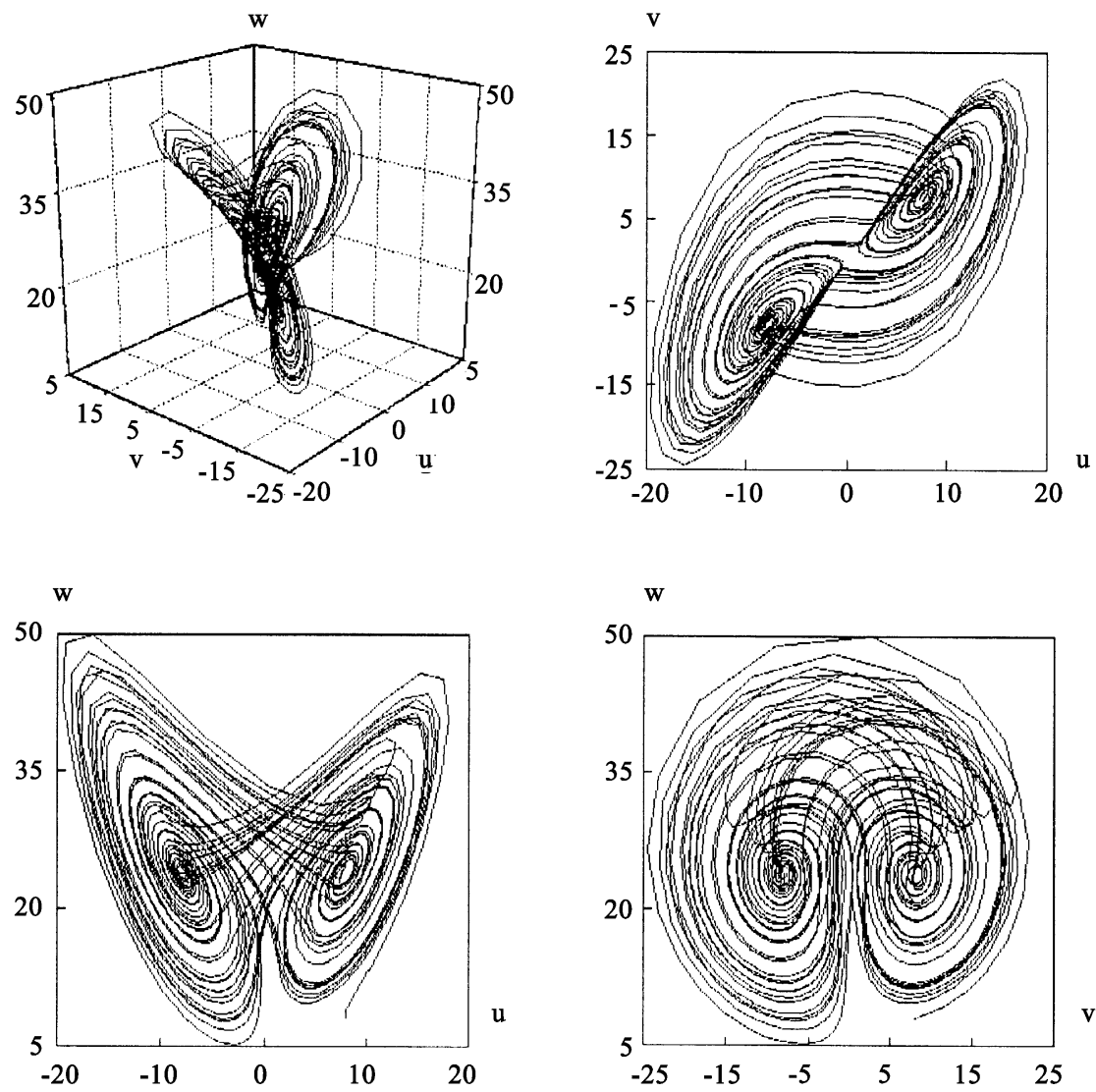

Fig. 2. Lorenz attractors of phase space at position $x=\frac{1}{2} N$. Parameters: $D_{1}=1.0 \times 10^{-2}, D_{2}=1.0 \times 10^{-2}, D_{3}=1.0 \times 10^{-2} ; \xi=10.0$, $\eta=24.8, \zeta=\frac{8}{3}, c=10.0,1 / \tau=2.2$. Initial conditions: $\left.u\right|_{t=0}=\left.v\right|_{t=0}=\left.w\right|_{t=0}=8$. . Lattice size: $N=200$. 

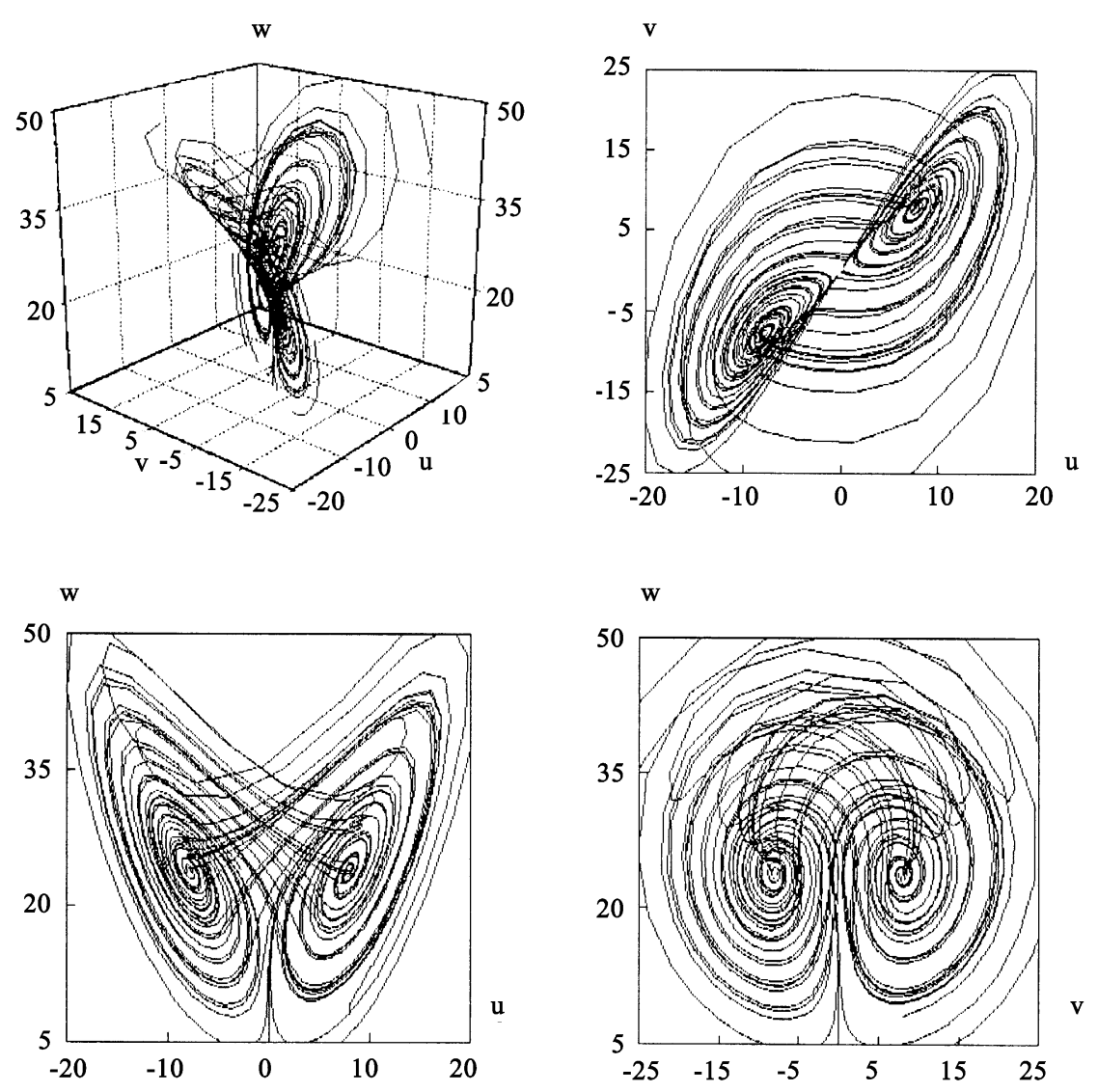

Fig. 3. Lorenz attractors of phase space at position $x=\frac{1}{2} N$. Parameters: $D_{1}=1.0, D_{2}=1.0, D_{3}=1.0 ; \xi=10.0, \eta=24.8, \zeta=\frac{8}{3}, c=10.0$, $1 / \tau=2.2$. Initial conditions: $\left.u\right|_{t=0}=\left.v\right|_{t=0}=\left.w\right|_{t=0}=8.1$. Lattice size: $N=200$.

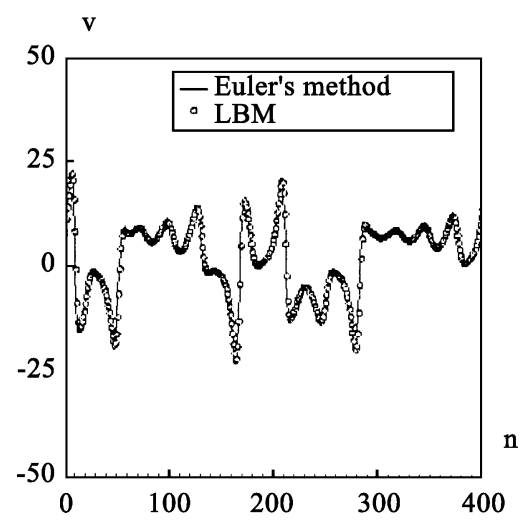

Fig. 4. Comparisons between LBM results and Euler method from 1 to 400 time steps at position $x=\frac{1}{2} N$. LBM (circle) and the Euler method (line) of $v$. Parameters: $D_{1}=1.0, D_{2}=1.0, D_{3}=1.0 ; \xi=10.0, \eta=24.8, \zeta=\frac{8}{3}, c=10.0,1 / \tau=2.2$. Initial conditions: $\left.u\right|_{t=0}=\left.v\right|_{t=0}=\left.w\right|_{t=0}=8$.1. Lattice size: $N=200$. 
2. $D_{\sigma}=1.0 \times 10^{-2}$.

3. $D_{\sigma}=1.0$.

For numerical simulation, we set a one-dimensional region $[0,1]$, with initial conditions

$$
u^{\sigma}=u_{0}^{\sigma}, \quad t=0
$$

and boundary conditions

$$
\frac{\partial u^{\sigma}(0, t)}{\partial x}=\frac{\partial u^{\sigma}(1, t)}{\partial x}=0 .
$$

In this paper, we set $\xi=10.0, \eta=24.8$ and $\zeta=\frac{8}{3}$ for the reaction terms, $c=10.0,1 / \tau=2.2$ for Eq. (2).

The additional terms are as follows:

$$
\phi^{1}=\frac{1}{\varepsilon(b+1)}(-\xi u+\xi v), \quad \phi^{2}=\frac{1}{\varepsilon(b+1)}(\eta u-v-u w), \quad \phi^{3}=\frac{1}{\varepsilon(b+1)}(-\zeta w+u v),
$$

and

$$
\lambda^{1}=\frac{D_{1}}{\varepsilon\left(\tau-\frac{1}{2}\right)}, \quad \lambda^{2}=\frac{D_{2}}{\varepsilon\left(\tau-\frac{1}{2}\right)}, \quad \lambda^{3}=\frac{D_{3}}{\varepsilon\left(\tau-\frac{1}{2}\right)} .
$$

We used $\left.u\right|_{t=0}=\left.v\right|_{t=0}=\left.w\right|_{t=0}=8.1$ as an initial condition and studied the trajectory in four phase spaces: $(u, v, w),(u, v),(u, w)$ and $(v, w)$. Figs. 1-3 show the calculated results plotted with 5000 points. It is seen that the Lorenz attractor appears. We then compared the numerical results of the LBM with the Euler method original 400 time steps. This can be observed in Fig. 4. The numerical result shows the phase picture as an attractor, and good agreement with existing results has been achieved.

\section{Conclusion}

In this paper, we have presented a new method for solving the Lorenz equation using the lattice BGK method. We obtained numerically the Lorenz attractor from $D_{\sigma}=0$ to 1 in this model. We are able to get more detailed results by numerical simulations. We only show three situations of $D_{\sigma}$. The result in $D_{\sigma}=0$ is very important, as it gives a new method for solution of ordinary differential equations. It is easy to construct other nonlinear chemical reaction systems by using higher moments of the lattice BGK model (e.g. the Belousov-Zhabotinski reaction and the Sel'kov reaction). As a numerical method, it may be considered as a tool to get patterns of some chemical reactions. We use an assumption in this model: $\omega_{\alpha}^{\sigma}=\varepsilon^{2} \phi_{\alpha}^{\sigma}$. Its meaning is that $D_{\sigma} \sim \psi^{\sigma}=\mathrm{O}\left(\varepsilon^{2}\right)$, i.e. $D a=\mathrm{O}(1)$ ( $D a$ is the unit Damkohler number). Till now, there is no method to remove this assumption in the field of the lattice BGK method. According to the linear stability of the difference equation, the stability of the lattice BGK schemes is based on the second viscosity, the third dispersion and the fourth viscosity. If we set $D_{\sigma}=0$, we only control the third coefficient of dispersion and the fourth coefficient of viscosity. We also select multi-speed lattice BGK model to give these coefficients if the macroscopic equation contains the third or the fourth coefficient [10]. These numerical results show that the assumption is reasonable. As a numerical method, the lattice BGK method may be not more efficient and more accurate standard ODE integration schemes. But we can find the ability of the lattice BGK method to simulate the nonlinear systems and the diffusion-reaction systems. It is interesting and difficult to compute the Lyapunov time scale of the Lorenz equations with the lattice BGK method. This problem will be explored in further research. 


\section{Acknowledgements}

We thank Prof. Z.S. She, Prof. Y.S. Chen, Prof. F.X. Zhou, Prof. S.X. Hu, Prof. X.Z. Jin and Prof. B.S. Wu for helpful discussions. This work is supported by National Key Program for Developing Basic Sciences (No. G1999032801) and the National Science Foundation of China, Grant No. 10072023.

\section{References}

[1] U. Frisch, B. Hasslacher, Y. Pomeau, Lattice-gas automata for the Navier-Stokes equations, Phys. Rev. Lett. 56 (1986) $1505-1508$.

[2] Y.H. Qian, D. d'Humiéres, P. Lallemand, Lattice BGK model for Navier-Stokes equation, Europhys. Lett. 17 (6) (1992) $479-484$.

[3] H.D. Chen, S.Y. Chen, M.H. Matthaeus, Recovery of the Navier-Stokes equations using a lattice Boltzmann method, Phys. Rev. A 45 (1992) 5339-5342.

[4] R. Benzi, S. Succi, M. Vergassola, The lattice Boltzmann equation: theory and applications, Phys. Rep. 222 (1992) $147-197$.

[5] S. Ponce Dawson, S. Chen, G.D. Doolen, Lattice Boltzmann computations for reaction-diffusion equations, J. Chem. Phys. 98 (2) (1993) 1514-1523.

[6] Y.H. Qian, S.A. Orszag, Scalings in diffusion-driven reaction A + B $\rightarrow$ C: numerical simulations by lattice BGK model, J. Stat. Phys. $81(1 / 2)(1995)$ 237-254.

[7] D. Wolf-Gladrow, A lattice Boltzmann equation for diffusion, J. Stat. Phys. 79 (5/6) (1995) 1023-1033.

[8] S. Succi, G. Bella, F. Papetti, Lattice kinetic theory for numerical combustion, J. Sci. Comput. 12 (4) (1997) $395-408$.

[9] O. Filippova, D. Haenl, Lattice BGK model for low-Mach combustion, Int. J. Mod. Phys. C 9 (8) (1998) 1439.

[10] G.W. Yan, Y.S. Chen, S.X. Hu, A lattice Boltzmann method for KdV equation, Acta Mech. Sinica 14 (1) (1998) $18-26$.

[11] G.W. Yan, Y.S. Chen, S.X. Hu, Simple lattice Boltzmann method for simulating flows with shock wave, Phys. Rev. E 59 (1999) $454-459$.

[12] S. Chapman, T.G. Cowling, The Mathematical Theory of Non-uniform Gases, Cambridge University Press, Cambridge, 1939.

[13] E.N. Lorenz, Deterministic non-periodic flow, J. Atmos. Sci. 20 (1963) 130-141.

[14] C. Sparrow, The Lorenz Equations, Springer, Berlin, 1982. 